\title{
¿Podemos favorecer el aprendizaje autónomo del significado de palabras?
}

\section{Ramiro Gilabert y Eduardo Vidal-Abarca}
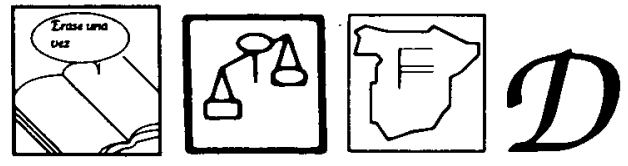

Se presenta aqui un programa de adquisición del vocabulario $y$ desarrollo de la comprensión a partir de contextos naturales. El programa, que abandona otras rutinas tradicionales de enseñanza de vocabulario, trata de mejorar la capacidad de inferencia $y$ comprensión textual de los alumnos para dotarles de mayor autonomía lectoescrita.

La enseñanza de vocabulario es considerada como una actuación tangencial o complementaria de otras actividades de comprensión. El reflejo de esta situación se manifiesta en los manuales de los cursos de enseñanza básica, pues presentan actividades de vocabulario muy generales que, a grandes rasgos, consisten en: a) apoyarse en los elementos morfológicos de las palabras; b) promover el uso del diccionario y c) proporcionar directamente el significado de las palabras.

Respecto a las actividades que utilizan los elementos morfológicos, la meta de las mismas es ayudar a la identificación del significado de las palabras a partir de los componentes internos de éstas. En otras palabras, se trata de facilitar el conocimiento de la información semántica que aportan los componentes estructurales de las palabras, también conocidos como claves internas (prefijos, sufijos, lexemas). En este bloque tienen cabida las actividades del tipo siguiente: formación de palabras nuevas a partir de su raíz: sal, sal-ero, sal-ina, sal-ado; comprensión del significado de prefijos (in-constante, des-hacer, re-pasar), e identificación del significado de palabras compuestas como lava-platos, sacacorchos.

El uso del diccionario también figura entre una de las actividades que se recomiendan para la adquisición del significado de las palabras. La tarea a desempeñar por e! alumno suele limitarse a: identificar el significado de una palabra mediante la pertinente consulta al diccionario, transcribir el significado correspondiente y usar éste a través de la producción oral o escrita. A pesar de la proliferación de este tipo de ejercicios, el diccionario no es el medio más eficaz 
para que los alumnos inexpertos adquieran el significado de las palabras (Anderson y Nagy, 1991). El tercer recurso que utilizan los libros de texto, consiste en suministrar directamente al alumno el significado de las palabras por medio de definiciones o glosarios.

Con esta primera aproximación quedaría dibujada una enseñanza tradicional del vocabulario. Aunque existen otras vías para incrementar el número de palabras conocidas (mapas semánticos, métodos orientados a la adquisición de conceptos, jerarquía de significados, "palabras-clave», etc.), las actividades descritas presentan la limitación de considerar las palabras aisladamente y de no ocuparse de promover entre los alumnos estrategias que ayuden a comprender el significado de las palabras.

Por otra parte, antes de que los niños reciban instrucción en vocabulario, ya conocen el significado de un amplio número de palabras (vocabulario pasivo), aunque usen un porcentaje reducido de éstas (vocabulario activo). Lo cual nos hace pensar que los alumnos aprenden significado de las palabras, sin que tenga que mediar necesariamente una instrucción explícita por parte del profesor. La pregunta a formular sería la siguiente: ¿a qué se deberá la adquisición de vocabulario sin haber recibido instrucción explícita? Ante la ausencia de argumentos de mayor entidad cabe aceptar la hipótesis de que es posible aprender de forma incidental el significado de palabras a partir del contexto oral o escrito. A pesar de la idea precedente, es notoria la ausencia de métodos que preparen a los niños en un mejor aprovechamiento del contexto. Sternberg y colaboradores (Sternberg, 1990; Sternberg y Powell, 1983) consideran importante la función que desempeña el contexto, y describen minuciosamente cómo utilizamos el contex to cuando nos hallamos ante una palabra cuyo sígnificado desconocemos. Para estos autores, los textos contienen una serie de indicios o pistas (claves contextuales externas) que nos informan sobre el significado de las palabras. En el texto de la Figura 1 nos encontramos con una palabra desconocida ("cornelo»), la cual está acompañada de información que en forma de pistas o claves contextuales externas, va indicándonos cuál es el significado de la palabra en cuestión. Estas claves nos hablan de: lugar donde se encuentra (1); origen (2); categoría a la que pertenece (3); causas que permiten encontrarlo (4); descripciones o características estáticas (5); usos o funciones que cumple (6); valor o importancia que posee (7) y (8); equivalencia con otros conceptos (9).

\section{FIGURA 1}

El «cornelo» se balla en (1) depósitos subterráneos a considerable profundidad. Su formación se ha debido a (2) los restos de seres vivos y vegetales que han quedado sepultados por grandes cantidades de tierra. Por sus características se le considera un (3) aceite mineral natural. Cuando se perfora (4) alguno de los citados depósitos, el «cornelo» que se extrae se balla en estado (5) líquido de color oscuro y con un intenso olor. Posteriormente es llevado a la refinería donde a partir de él, se obtendrán (6) otros productos (gasolina, aceite, gas) que son muy necesarios para que (7) funcionen los coches, fábricas, calefacciones, etc. El «cornelo» es muy importante para (8) la vida de los pueblos por la riqueza que genera como lo demuestra la gran cantidad de historias, leyendas y películas que tienen al oro negro como protagonista central (9).

Modelo de texto en el que se ejemplifican algunas de las claves textuales señaladas por Stermberg y colaboradores 


\section{FUNDAMENTACION DE LA INSTRUCCION EN VOCABULARIO}

La enseñanza tradicional de vocabulario recibe diversas críticas porque se basa en actuaciones aisladas y fuera de contexto. En otro sentido, un método de instrucción de vocabulario será eficaz en la medida en que proporcione «definiciones adecuadas y ejemplos precisos de cómo son usadas las palabras en los contextos naturales» (Nagy, 1988, pág. 9). Si además agregamos que un método eficaz también debe destacar por su capacidad de incrementar el nivel de comprensión lectora, quedaría definido el modelo de método ideal. Este autor propone los siguientes rasgos del método ideal:

a) Capacidad de integración de las palabras instruidas. Las palabras objeto de instrucción serán susceptibles de relación o integración con otros conocimientos del sujeto. En este principio se materializa la teoría de los esquemas al propugnar la estructuración del conocimiento, así como el reconocimiento de que cualquier nueva adquisición de información está influida por el conocimiento previo.

b) Posibilidad de repetición de las palabras. El hecho de encontrarse varias veces con la misma palabra facilita su identificación, con la consiguiente economía de recursos cognitivos, consiguiendo un acceso al significado rápido y eficaz.

c) Uso significativo de las palabras aprendidas. Este rasgo descansa en los siguientes principios: implicación activa de los sujetos para conseguir más aprendizaje, que los sujetos piensen sobre las palabras objeto de aprendizaje para aumentar la profundidad de procesamiento y el recuerdo, y que exista la posibilidad de usar contextualmente las palabras aprendidas.

El programa que presentamos pretende enseñar estrategias para que los sujetos comprendan mejor las palabras desconocidas que aparecen en los textos. Realmente se trata de mejorar la competencia en inferencia del significado de las palabras, y conseguir que los lectores sean más autónomos a la hora de comprender las palabras que encuentran mientras leen. El programa ha sido aplicado y probada su eficacia en situaciones controladas (Gilabert, 1990) y en ambientes escolares ordinarios correspondiendo la aplicación a los propios tutores de los alumnos (Gilabert y Vidal-Abarca, 1989). En este programa recogemos algunas de las sugerencias anteriores (Nagy, o.c.), así como otros rasgos relevantes que enumeramos:

1. El alumno se apoya en un heurístico que le ayuda en la identificación del significado de las palabras, a través de un conjunto de estrategias de aprendizaje. Estas estrategias están supeditadas, a su vez, a un procedimiento de enseñanza aprendizaje que armoniza los postulados de la instrucción directa (Baumann, 1985) y los de la enseñanza recíproca (Palincsar y Brown, 1984).

2. Las estrategias de aprendizaje (Nisbet y Shucksmith, 1987) que recoge el heurístico permiten: la formulación de cuestiones previas (clarificar los fines de la tarea, descomponer la tarea en sus diferentes componentes); la planificación y el control (establecer la táctica correspondiente) y la comprobación de la respuesta (autoevaluación y revisión).

3. El programa posee un nivel alto de contextualización, pues admite ser aplicado con textos de los alumnos e integrado en las actividades ordinarias del aula. En nuestro caso lo hicimos en situaciones educativas reales, como una actividad más de la asignatura de lenguaje.

4. El programa favorece la conexión entre el significado de la palabra que hay que identificar y los conocimientos previos del sujeto, con objeto de que 
la nueva información pueda ser integrada en las estructuras de conocimiento del sujeto.

5. La figura del maestro es fundamental por cuanto dirige el proceso en todas sus fases y porque proporciona la oportuna retroalimentación.

\section{EL PROGRAMA DE INSTRUCCION}

El programa constaba de 18 sesiones, las cuales tenían una duración máxima de 40 minutos. Semanalmente realizábamos dos sesiones, en horario lectivo, y el desarrollo de las mismas estaba dirigido por uno de los autores de este trabajo, en presencia del tutor del aula. El programa de instrucción ha sido utilizado en repetidas ocasiones con alumnos desde $30^{\circ}$ a $5 .^{\circ}$ de EGB, aunque variando los textos para los sujetos más pequeños.

Los textos del programa pertenecían a las áreas de Ciencias Sociales y Naturales, realizándose en ellos algunas modificaciones como: sustituir en cada texto una palabra por otra «palabra misteriosa» (pseudopalabra) cuya frecuencia de aparición variaba; controlar el tipo de claves contextuales y la distancia entre éstas y la palabra a la que se referían. Con los textos establecimos dos categorías: textos de nivel explícito y textos de nivel implícito. La diferencia básica entre estos textos residía en que los primeros contenían claves contextuales muy evidentes o claramente manifiestas, mientras que los segundos se distinguían por presentar claves contextuales poco evidentes o sobreentendidas.

El desarrollo del programa se fundamentaba en un heurístico o procedimiento que tenía como misión la de guiar a los alumnos en la inferencia del significado de las palabras desconocidas. El heurístico constaba de una serie de estrategias, ya indicadas en el apartado anterior. Asimismo, a partir de los textos formulábamos ejercicios que tenían como objetivo la activación de las estrategias de inferencia. También distinguimos cuatro etapas en la aplicación del programa: introducción, de significado explícito, de significado implícito y combinada (Figura 2). Esta diferenciación en etapas permitía mantener la unidad del programa y, simultáneamente, establecer fases en cada una de ellas, de acuerdo con las orientaciones de la instrucción directa y que detallaremos a continuación.

En la etapa de introducción informábamos de cuál era la finalidad del programa, resaltando los beneficios que éste generaría. Al mismo tiempo realizábamos actividades colectivas de inferencia del significado. Con éstas deseábamos que los alumnos tomaran conciencia que el significado de una palabra, depende tanto de la información textual como de la construcción que hace el propio lector. Iniciábamos el proceso presentando gradualmente, a través de una transparencia, un texto mutilado (Figura 3). La tarea de los alumnos consistía en avanzar y justificar el posible significado de la palabra ausente, conforme leían el texto. Como la identificación del significado solicitado no podía conseguirse hasta la lectura total del texto, los alumnos se percataban de: el carácter erróneo de las hipótesis iniciales, cómo contribuían las claves textuales a la inferencia del significado y cómo éste dependía de la construcción que hiciera el propio lector.

En la etapa de significado explícito (sesiones $2 .^{\mathrm{a}}$ a $4 .^{\mathrm{a}}$ ) los textos poseían las claves explícitas, siendo escasa la distancia entre éstas y la palabra desconocida a la que acompañaban. En primer lugar los sujetos observaban la aplicación del heurístico para averiguar el significado de palabras desconocidas (fase 
FIGURA 2

\begin{tabular}{|c|c|c|c|}
\hline Etapa instruccional & Sesiones & Objetivos & Fase \\
\hline Instrucción & 1 & $\begin{array}{l}\text { Informar del objetivo } \\
\text { del programa y motivar } \\
\text { en el uso de las } \\
\text { inferencias }\end{array}$ & \\
\hline \multirow[t]{4}{*}{$\begin{array}{l}\text { Significado } \\
\text { explícito }\end{array}$} & 2 & $\begin{array}{l}\text { Identificación claves } \\
\text { textuales explícitas }\end{array}$ & Modelado \\
\hline & 3 & & Instrucción directa \\
\hline & $4 \cdot 5$ & & Instrucción dirigida \\
\hline & $6-7$ & & Trabajo autónomo \\
\hline \multirow[t]{2}{*}{$\begin{array}{l}\text { Significado } \\
\text { implícito }\end{array}$} & $8-9$ & $\begin{array}{l}\text { Identificación claves } \\
\text { textuales implícitas }\end{array}$ & Instrucción directa \\
\hline & 10 & & Instrucción dirigida \\
\hline Combinada & $\begin{array}{l}11-14 \\
15-18\end{array}$ & $\begin{array}{l}\text { Identificación claves } \\
\text { textuales explícitas e } \\
\text { implícitas }\end{array}$ & $\begin{array}{l}\text { Trabajo autónomo } \\
\text { Trabajo autónomo }\end{array}$ \\
\hline
\end{tabular}

Características generales del programa de instrucción.

FIGURA 3

«Nada más sentarme en la mesa me doy cuenta que he olvidado el y me es imposible hacer los ejercicios de matemáticas.»

- ¿Qué he olvidado? ¿Por qué?

- ¿Qué otras cosas puede haber olvidado?

«Si le pido a mi compañera que me lo preste, ella no podrá trabajar tampoco puesto que solamente tiene uno.»

- ¿De qué cosa se trata? ¿Por qué?

- ¿En qué lugar se encuentra? ¿Por qué?

- ¿Qué palabra te ha dado la pista del lugar donde está?

«Ya sé lo que puedo hacer. Colocaré la mesa junto a la suya y de este modo leeremos los dos.»

- ¿Sabes cuál es el objeto que ha olvidado? ¿Por qué?

- ¿Qué palabra te ha dado alguna pista?

- El que ha olvidado el objeto, ¿es chico o chica? ¿Por qué?

de modelado). En este proceso de modelado el instructor acompañaba cada una de las actividades con verbalizaciones específicas que se referían a la explicación o justificación de lo que hacía, dudas que surgían durante el proceso, hipótesis aproximativas que formulaba, etc. Así, mientras el alumno observaba lo que hacía el instructor, éste iba diciendo en voz alta: «En el párrafo primero se dice de las salistas ... tierras bajas que son inundadas por el agua del mar; ... reciben el nombre de salistas. En el siguiente párrafo se indica que ... En el últi- 
mo ...» (Figura 4). Una vez los alumnos conocían en qué consistía la tarea, éstos comenzaban a cumplimentar las actividades propuestas bajo la dirección y control del instructor. Seguidamente cada alumno llevaba a la práctica las conductas observadas previamente, y así adquirir los conocimientos precisos para aplicar los pasos de que constaba el heurístico (fase de instrucción directa). Una vez conseguida esta meta, los alumnos volvían a realizar actividades similares a las anteriores, mientras que el instructor sólo intervenía si era requerido por los alumnos por alguna duda u obstáculo (fase de instrucción dirigida). Finalmente cada alumno realizaba por su cuenta las actividades de la sesión y posteriormente tenía lugar una discusión con participación de toda la clase (fase de trabajo autónomo).

Figura 4

SESION 1

Significado explícito

MODELADO

EL TRABAJO QUE VAS A COMENZAR ESTA DESTINADO A QUE APRENDAS EL SIGNIFICADO DE LAS PALABRAS

PRESTA ATENCION A COMO TU PROFESOR ENCUENTRA EL SIGNIFICADO DE LAS PALABRAS DESCONOCIDAS

TEXTO N..$^{\circ}$

TEXTO N: 1

Cerca de la costa y de la desembocadura de los ríos hay

1..$^{\circ}$ tierras bajas que son inundadas por el agua del mar. Estas tierras reciben el nombre de «salistas».

2. $[$ El agua de las «salistas» es salada y las plantas que en ellas crecen son de pequeño tamaño.

Estos parajes son habitados por aves que después de un

3..$^{\circ}$ tiempo se marchan. Otros animales que también viven en las «salistas» son los cangrejos, almejas y caracoles.

RECUERDA TODO LO QUE TIENES QUE HACER PARA ENCONTRAR LA PALABRA MISTERIOSA

1. Lee todo el texto

$1 '$ 2. Fíjate si existe alguna palabra que no sabes lo que significa.

3. Busca el significado de esa palabra en cada uno de los párrafos.

4. Comprueba si el significado encontrado es correcto.

Sesión segunda, utilizada como modelo para la enseñanza del heurístico de inferencia del significado explicito.

La etapa de significado implícito (sesiones $8 .^{\mathrm{a}}$ a $14 .^{\mathrm{a}}$ ) constaba de textos con pistas o claves textuales poco evidentes y éstas no se hallaban cerca de la palabra a la que definían. Los textos también eran de mayor extensión, en general, que los de la etapa anterior. Como los sujetos ya estaban familiarizados con el heurístico, iniciábamos esta etapa con la fase de instrucción directa y seguíamos un proceso idéntico al descrito en la etapa precedente, excepto en la importancia concedida a la discusión colectiva. Aunque en la fase de trabajo autónomo 
cada alumno ejecutaba de forma individual los ejercicios propuestos, en todas las sesiones destinábamos un tiempo a la discusión colectiva de acuerdo con la siguiente organización: un alumno formulaba oralmente la respuesta, así como la justificación correspondiente; el resto de compañeros contrastaba la anterior respuesta con la suya propia, recogiéndose aquellas aportaciones que mejoraban la respuesta inicial; en el caso de las respuestas erróneas, otros compañeros asumían la responsabilidad de explicar el error y proponer y justificar una respuesta alternativa correcta. La secuencia de la discusión estaba marcada por el orden de las actividades de la sesión correspondiente (Figura 5).

FIGURA 5

\section{SESION 8}

\section{Significado implícito}

\section{INSTRUCCION DIRECTA}

1. LEE el texto $\mathrm{n}^{\circ} 13$ y FIJATE si hay alguna palabra desconocida.

Los huesos sostienen nuestro cuerpo y le dan forma. Sin ellos no podríamos estar de pie, ni menos aún trasladarnos de un lugar a otro. Además, también evitan que los órganos importantes como el corazón o los pulmones reciban golpes, ya que las costillas les protegen.

Los huesos se encuentran unos junto a otros, aunque no siempre de la misma manera. Hay «infelaciones» móviles y fijas.

En las «infelaciones» móviles los huesos casi no se tocan y se mueven como ocurre con los huesos que se encuentran en la rodilla o el codo.

Sin embargo, en las «infelaciones» fijas los huesos se hallan siempre en contacto y no se mueven. Un ejemplo lo tenemos en los huesos de la cabeza que están fuertemente soldados entre sí.

Resumiendo, si las uniones de los huesos son móviles podemos realizar los más variados movimientos, pero si son fijas, no podemos hacer ninguna clase de movimiento pues los huesos se hallan completamente inmóviles.

2. ¿Qué dice el primer párrafo sobre las «infelaciones»?

3. ¿Qué información aparece en el segundo párrafo referida las «infelaciones»?

4. ¿Hay más información en el tercer párrafo? ¿Cuál?

5. ¿Y en el cuarto párrafo?

6. ¿Y en el último párrafo?

7. RECUERDA todo lo que sabes sobre las «infelaciones» y COMPLETA:

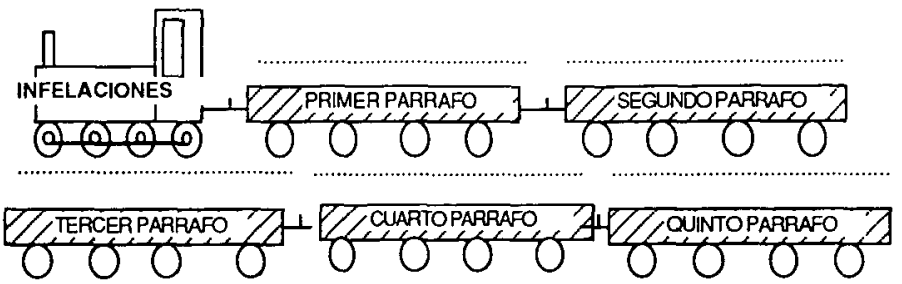

8. Formula con tus propias palabras el significado de «infelaciones»: ............

Modelo de sesión abreviada de la etapa de significado implícito.

Por último, en la etapa combinada (sesiones $15^{\mathrm{a}}$ a $18 .^{\mathrm{a}}$ ) trabajábamos con textos de significado explícito e implícito. Todas las sesiones estuvieron destinadas al trabajo autónomo, como puede observarse en la Figura 2. La discusión colectiva la ocupábamos en la evaluación de las respuestas, tal como hemos avan- 
zado, y, sobre todo, en la identificación de las claves textuales que apoyaban las respuestas.

Un aspecto pendiente de comentario es el referido al tipo de actividades que integraban el programa. Las actividades fundamentales eran las específicas del heurístico, las cuales estaban orientadas a la identificación del significado de las palabras desconocidas. Estas actividades aparecían en cada una de las sesiones, y resumidamente consistían en: lectura del texto por párrafos; identificar (escribir o subrayar) la «palabra misteriosa» que contenía cada uno de los textos; localizar en cada texto las claves contextuales que aportaban información sobre la «palabra misteriosa»; comparar las claves que contenían los distintos párrafos, justificando semejanzas y diferencias; resumir la información obtenida y producir con las propias palabras del alumno una definición de la «palabra misteriosa» (Figura 5). Con este procedimiento, aunque no garantizábamos que todos los sujetos alcanzasen el éxito en la tarea, sí que proporcionábamos a éstos un instrumento para que pudieran conseguirlo. Otro grupo de ejercicios estaba constituido por actividades de tipo inferencial, similares a las descritas en la etapa de introducción (Figura 3).

\section{REPERCUSIONES INSTRUCCIONALES}

Muchos métodos de instrucción en vocabulario pierden parte de su validez por presentar algunas insuficiencias como: son actuaciones puntuales sin carácter de continuidad, se centran en la enseñanza del significado de un número limitado de palabras, es problemática su generalización a los ambientes escolares ordinarios, precisan de materiales específicos, etc.

El programa descrito ofrece vías de solución para algunas de las insuficiencias acabadas de enunciar. En primer lugar el programa es susceptible de ser aplicado en ambientes educativos ordinarios, como si se tratase de una actividad más del currículum escolar. En nuestro caso lo hacíamos compartiendo el tiempo destinado al área de lenguaje. En segundo lugar, el programa admite un alto grado de contextualización puesto que los textos a utilizar pueden ser los mismos que los que usa el alumno habitualmente. Para disponer de los textos del alumno sólo se precisa sustituir la palabra cuyo significado se desea instruir por una pseudopalabra y seguir las recomendaciones de aplicación del programa. Finalmente y para evitar que la aplicación del programa se convierta en una actuación puntual, se requerirá que el mismo sea utilizado en las restantes áreas de contenido. En el caso de que los alumnos ya estén familiarizados con la aplicación del heurístico, cuando aparezcan palabras desconocidas (o conceptos) en los textos de trabajo, se aprovechará la ocasión para que los alumnos hagan uso de las pertinentes estrategias de inferencia. De este modo mejoraríamos la comprensión y aprendizaje de vocabulario, así como la eficacia del estudio de los alumnos.

\section{Referencias}

Anderson, R. C. y NaGy, W. E. (1991). Words Meanings. En R. Barr, M. L. Kamil, P. B. Mosenthal y P. D. Pearson (Eds.), Handbook of Reading Research. Vol. II. Nueva York: Longman.

BaUmann, J. F. (1985). La eficacia de un modelo de instrucción directa en la enseñanza de la comprensión de ideas principales. Infancia y Aprendizaje, 31-31, 89-105. 
GILABERT, R. (1990). La instrucción del significado por el contexto, en la lectura comprensiva en el Ciclo Medio de E. G. B. Tesis Doctoral. Universidad de Valencia.

Gilabert, R. y VIDAl-AbarCa, E. (1989). Comprender para aprender: un proyecto de innovación educativa. Proyecto subvencionado por la Conselleria de Cultura, Educació y Ciència de la la Generalitat Valenciana.

$\mathrm{N}_{\mathrm{AGY}}, \mathrm{W}$. E. (1988). Teaching vocabulary to improve reading comprehension. Newark, Delaware: International Reading Association.

Nisbet, J. y Shucksmith. J. (1987). Estrategias de aprendizaje. Madrid: Santillana.

Palincsar, A. S. y Brown, A. L. (1984). Reciprocal teaching of comprehension-fostering and comprehension-monitoring activities. Cognition and Instruction, 1 (2), 117-175.

StERnBerg, R. J. (1990). Más allá del cociente intelectual. Bilbao: Editorial Desclee de Brouwer.

Sternaerg, R. J. y Powell, J. S. (1983). Comprehending verbal comprehension. American Psychologist, August, 878-893.

\section{¿Podemos favorecer el aprendizaje autónomo del significado de palabras? Ramiro Gilabert Pérez y Eduardo Vidal-Abarca Gámez. CL\&E, 1993, 17, pp. 81-89}

Resumen: La instrucción en vocabulario es comtemplada por los distintos manuales como complemento de otras actividades. Esto representa que los alumnos no reciban una instrucción que les ayude a comprender mejor las palabras. El programa que presentamos posibilita que los alumnos comprendan de forma autónoma el significado de las palabras. Otro rasgo innovador del programa es que puede ser aplicado por los tutores del aula, dentro del horario lectivo y utilizar textos de las distintas áreas con que trabajan habitualmente los niños.

Datos sobre los autores: Los autores poseen experiencia en el campo de la educación, primero como maestros especialistas en el tratamiento de dificultades de aprendizaje, y en la actualidad como profesores de E.U. de Formación del Profesorado. Recientemente han publicado trabajos que tratan sobre aspectos teórico-prácticos de la comprensión y el vocabulario.

Dirección: Universidad de Valencia. E.U. de Formación del Profesorado de EGB Alcalde Reig, 8. 46006 Valencia.

(C) De todos los artículos deberá solicitarse por escrito autorización de CL\&E y de los autores para el uso en forma de facsímil, fotocopia o cualquier otro medio de reproducción impresa. CL\&E se reserva el derecho de interponer las acciones legales necesarias en aquellos casos en que se contravenga la ley de derechos de autor. 